Research Paper

\title{
Comparative safety and effectiveness of ultrasound-guided radiofrequency ablation combined with preoperative three-dimensional reconstruction versus surgical resection for solitary hepatocellular carcinoma of $3-5 \mathrm{~cm}$
}

Yuanfeng Gong ${ }^{1,2^{*}}$, Yunqiang Tang ${ }^{2 *}$, Yan Geng1,3, Yu Zhou ${ }^{1}$, Min Yu' ${ }^{1}$, Bowen Huang ${ }^{1}$, Zhonghai Sun ${ }^{1}$, Hui Tang ${ }^{2}$, Zhixiang Jian' ${ }^{1}$, Baohua Hou ${ }^{\circledR}$

1. Department of General Surgery, Guangdong Provincial People's Hospital, Guangdong Academy of Medical Sciences, No.106 Zhongshan 2nd Rd, Yuexiu Dist, Guangzhou 510080, China;

2. Department of Hepatobiliary Surgery, the Affiliated Cancer Hospital \& Institute of Guangzhou Medical University, No.78 Hengzhigang Rd, Yuexiu Dist, Guangzhou 510095, China;

3. Department of Gastrointestinal Surgery, Shunde Hospital of Southern Medical University, No.1 Jiazi Rd, Shunde Dist, Foshan 528300, China.

* These authors contributed equally to this work.

$\square$ Corresponding author: Baohua Hou, Department of General Surgery, Guangdong Provincial People's Hospital, Guangdong Academy of Medical Sciences, No.106 Zhongshan 2nd Rd, Yuexiu Dist, Guangzhou 510080, China; houbaohuahbp@163.com; Tel.: +86 2083827812

(1) The author(s). This is an open access article distributed under the terms of the Creative Commons Attribution License (https://creativecommons.org/licenses/by/4.0/). See http://ivyspring.com/terms for full terms and conditions.

Received: 2018.12.17; Accepted: 2019.05.04; Published: 2019.09.03

\begin{abstract}
Objective: To investigate the safety and effectiveness of ultrasound-guided radiofrequency ablation (RFA) combined with preoperative three-dimensional (3D) reconstruction versus surgical resection for solitary hepatocellular carcinoma of 3-5 cm.

Methods: The cohort of this retrospective study included 66 consecutive patients who underwent open hepatectomy (Surgery group) between January 2009 and December 2014, as well as 54 consecutive patients who underwent ultrasound-guided RFA combined with preoperative 3D reconstruction (RFA group) during the same period. Preoperative 3D reconstruction was performed using Myrian-XP-Liver software. The image fusion system was used to evaluate the RFA safety margin at 1 month after surgery. Kaplan-Meier analysis and the log-rank test were used to compare the recurrence and overall survival (OS) rates between the two treatment groups.

Results: There were no significant differences in the baseline characteristics of the two groups. The complete ablation rate was $94.4 \%$ (51/53). As compared with surgical resection for solitary HCC of 3-5 cm, ultrasound-guided RFA combined with preoperative 3D reconstruction significantly reduced the morbidity of excessive pain, total complications, and infections $(p<0.001)$. A significant decrease in the duration of the hospital stay after treatment was also observed in the RFA group ( $t$ $=10.017, p<0.001)$. There was no significant difference in the cumulative recurrence rate between the two groups. Kaplan-Meier analysis and the log-rank test revealed no significant difference in the OS rate between the two groups over a 3-year follow-up period.

Conclusion: Ultrasound-guided RFA combined with preoperative 3D reconstruction appears to be a safe and effective therapeutic option for patients with solitary $\mathrm{HCC}$ of $3-5 \mathrm{~cm}$.
\end{abstract}

Key words: hepatocellular carcinoma, radiofrequency ablation, three-dimensional reconstruction, threedimensional image fusion, hepatectomy 


\section{Introduction}

Hepatocellular carcinoma (HCC) is the sixth most common cancer and the second most common cause of cancer-related death worldwide, accounting for an estimated 782,500 new cases and 745,500 deaths annually [1]. Although liver resection is the primary treatment for HCC, extensive surgical resection has certain limitations due to the risk of liver dysfunction after ischemia-reperfusion injury, the inflammatory response, nutrient metabolism abnormalities, and differences in liver cirrhosis status and resection range [2]. In recent years, ultrasound-guided radiofrequency ablation (RFA) of small HCC has been widely applied in clinical practice. For lesions smaller than $3 \mathrm{~cm}$, RFA has the same safety and effectiveness as surgical resection [3-4], while for lesions with a diameter of 3-5 cm, the curative effect remains controversial [5]. Incomplete ablation is the main factor that affects its curative effect. Accurate preoperative imaging assessment can not only reduce the complication rate of RFA, but also ensure a tumor-free margin and improve survival [6]. Preoperative three-dimensional (3D) reconstruction is used to stereoscopically display the tumor location, intrahepatic vasculature, and adjacent organs to better plan and improve the safety and effectiveness of RFA [7-8]. Thus, the aim of this retrospective study was to comprehensively investigate the safety and effectiveness of ultrasound-guided RFA combined with preoperative $3 \mathrm{D}$ reconstruction versus surgical resection for HCC of 3-5 cm.

\section{Materials and Methods}

\section{Subjects}

The cohort of this retrospective study included 66 consecutive patients who underwent open hepatectomy between January 2009 and December 2014, as well as 54 consecutive patients who underwent ultrasound-guided RFA combined with preoperative $3 \mathrm{D}$ reconstruction during the same period. The study protocol was approved by the Ethics Committee of the Hospital and written informed consent was obtained directly from all participants. All subjects met the following criteria: (1) hepatectomy or RFA as the first-line treatment, but no previous chemotherapy or radiotherapy; (2) confirmed diagnosis of HCC by history, AFP level, image study, and postoperative pathological analysis; and (3) Child-Pugh grade A or B, with one tumor with a diameter of 3-5 cm, with no macrovascular invasion or distant metastasis. Exclusion criteria were as follows: (1) unavailability of pre- and post-operative computed tomography (CT) data, as some patients underwent preoperative enhanced CT in other hospitals and we could not obtain the digital CT data. (2) loss to follow-up within 3 months after RFA or surgery, (3) any other malignancy, or (4) the anatomical structure around the ablation area was obviously changed (e.g., massive ascites), which might affect the fusion of images.

\section{D reconstruction and RFA procedure}

Preoperative enhanced CT was performed using the GE Lightspeed 64-slice helical CT system (GE Healthcare Life Sciences, Chicago, IL, USA). MyrianXP-Liver software (Intrasense SA, Montpellier, France) was used for 3D reconstruction. Ultrasoundguided (Hitachi Aloka SSD-Alpha 5 Surgical Ultrasound System; Hitachi Ltd., Tokyo, Japan) percutaneous RFA was conducted with the Cool-tip RFA System (Medtronic plc, Dublin, Ireland) with a 17G electrode needle. Percutaneous liver biopsy was performed using a 16G Tru-Core needle $(15 \mathrm{~cm}$; Angiotech, Wheeling, IL, USA).

For CT assessment, all patients were placed in the supine position. The scanning area ranged from the diaphragm to the kidney. The scanning parameters were as follow: current, $250 \mathrm{~mA}$; voltage, $120 \mathrm{kV}$; slice thickness, $5 \mathrm{~mm}$; pitch, 0.984; and rotation speed, $0.5 \mathrm{~s} /$ cycle. The contrast agent was highly concentrated Ultravist 370 (Bayer Pharma AG, Wuppertal, Germany). The scanning was delayed 20-25 s after the arterial phase and 55-60 s after the venous phase. CT data of the arterial and venous phases, as well as the portal vein phase were transferred to the GE AW4.4 workstation, copied onto a DVD (DICOM format), and subsequently imported into Myrian-Xp-Live software. The system automatically identified and extracted image information of the liver parenchyma, tumors, hepatic artery, portal vein, and hepatic vein, and then generated reconstructed $3 \mathrm{D}$ images of the liver. The tumor location and size, as well as the adjacent vasculature could be clearly observed in all directions. Sometimes, the automatic identification was insufficient or excessive, thus appropriate cutting was manually performed. The liver volume was estimated with Myrian-Xp-Live software.

RFA procedure: The 3D RFA target area (a tumor-free margin of at least $5 \mathrm{~mm}$ ) was designed according the preoperative $3 \mathrm{D}$ reconstructed image. It was not possible to reach such a safety margin in some cases owing to the adjacent vasculature, intestines, or gallbladder. Under local or general anesthesia, ultrasound-guided percutaneous liver biopsy was performed. Then, a 17G Cool-tip electrode needle was punctured into the liver under real-time ultrasound guidance. The radiofrequency power was gradually increased and lasted about $10 \mathrm{~min}$. The puncture track 
was ablated once the procedure was finished in order to reduce postoperative bleeding and seeding of tumor cells. Sometimes, the electrode needle could not be accurately inserted when the tumor was located near the hepatic dome due to lower chest interference. For such cases, the artificial hydrothorax technique was applied by injecting $800-1000 \mathrm{~mL}$ of saline into the right thoracic cavity to push the diaphragm downwards. After the RFA procedure, Doppler ultrasound was used to detect the target area and determine whether the blood flow signal had still existed.

\section{Effect evaluation}

Definitions of postoperative complications were as follows: Post-hepatectomy hemorrhage was defined as a decrease in postoperative hemoglobin levels of $>3 \mathrm{~g} / \mathrm{dL}$, as compared with the baseline level and/or any postoperative transfusion of packed red blood cells and/or the need for radiological intervention (such as embolization), and/or re-operation to stop the bleeding. Evidence of intra-abdominal bleeding was obtained by imaging or blood loss via the abdominal drains, if present. Biliary leakage was defined as a drainage bilirubin to serum bilirubin ratio of $>3$ at day 3 or after resection, or interventional surgical revision due to biliary peritonitis. Post-hepatectomy liver failure was defined as an increase in the international normalized ratio and hyperbilirubinemia on or after day 5. A clinical diagnosis of sepsis was defined as systemic inflammatory response syndrome with a documented infection. Systemic inflammatory response syndrome was demonstrated by two or more of the following four variables: (1) hyperthermia $\left(>38.3^{\circ} \mathrm{C}\right)$ or hypothermia $\left(<36^{\circ} \mathrm{C}\right) ;$ (2) tachycardia $(>90$ beats/min); (3) tachypnea (rate $\geq 20$ breaths/min) or hypoxia (oxygen saturation $<90 \%$ or need for oxygen supplementation of $\geq 0.4$ fraction of inspired oxygen to maintain adequate saturation); and (4) leukocytosis [white blood cell (WBC) count of $>12.0 \times 10^{9}$ cells/L], leukopenia (WBC count of $<4 \times 10^{9}$ cells/L), or immature or band forms of $>10 \%$. Postoperative ileus was defined as two or more of the following symptoms: nausea/vomiting, inability to tolerate an oral diet for more than $24 \mathrm{~h}$, absence of flatus for more than $24 \mathrm{~h}$, distension, and radiological confirmation on or after day 4 without prior resolution of ileus.

An increase in the WBC count was induced by the inflammatory reaction. Pre- and post-operative liver function was assessed using the Child-Pugh scoring system based on serum levels of alanine aminotransferase (ALT), aspartate transaminase (AST), total bilirubin (TBil), and albumin (Alb), as well as the prothrombin time (PT). Preoperative reserved liver function was measured according to the indocyanine green retention rate at $15 \mathrm{~min}$ (ICG15). Venous blood samples were obtained from all subjects before surgery and on postoperative days 1 (D1), 3 (D3), and 7 (D7). The preoperative characteristics of all patients [i.e., age, sex, body mass index, fasting blood glucose, hepatitis B surface antigen (HBsAg) status, Child-Pugh score, ICG15, and alphafetoprotein (AFP)], intraoperative characteristics (surgical duration, complete ablation rate, blood loss, and Pringle time), and postoperative characteristics (excessive pain, duration of postoperative hospital stay, complications, and mortality) were collected.

An image fusion system (Myrian-Xp-Live software), as described in our previous study [9], was used to evaluate the RFA safety margin at 1 month after surgery. Images of the ablation area and tumor were superimposed in a 3D model (Figure 1). The transparency of two models (per- and post-RFA) were adjusted and rotated to determine and measure the shortest tumor-free margin. A safety margin of $5 \mathrm{~mm}$ or more was considered as complete ablation, while less than $5 \mathrm{~mm}$ was considered as incomplete ablation [9].

\section{Follow-up}

Tumor recurrence was diagnosed by enhanced $\mathrm{CT}$ and/or magnetic resonance imaging (MRI). Liver biopsies of new lesions were unnecessary. CT and/or MRI were performed at 4 weeks after treatment and every 2 month thereafter during the first 2 years. At each of these follow-up visits, blood tests including liver function tests and serum AFP were done. Chest radiography was done every 6 months. The follow-up visits were spaced out to once every 3 months after 2 years. Tumor recurrence was defined as a new enhanced mass in the arterial phase with washout in the portal site or a delayed phase. A mass showing arterial enhancement without washout was considered as a progressively growing mass on follow-up images or lipiodol retention after transcatheter arterial chemoembolization (TACE) as recurrent HCC. TACE or RFA was performed in the case of neoplasm recurrence. The recurrence time was calculated from the end of surgery to the diagnosis of tumor recurrence. The overall survival (OS) rate was calculated from the end of surgery to the time of death or the latest follow-up. The postoperative 1-, 2-, and 3-year progression-free survival (PFS) and OS rates were compared between the RFA and surgery groups.

\section{Statistical Analysis}

Continuous variables are presented as the mean and standard deviation. The Student's $t$-test was used to identify differences between the means of two 
continuous variables. The $\chi^{2}$ test or Fisher's exact probability test was used to determine whether the frequencies between the RFA and surgery groups were significantly different $(\alpha=0.05)$. To evaluate the long-term effect of ultrasound-guided RFA combined with preoperative $3 \mathrm{D}$ reconstruction, the prognoses between the RFA and surgery groups were compared. The recurrence and OS rates were calculated by the Kaplan-Meier method. The survival curves were plotted by Kaplan-Meier analysis and compared by the log-rank test. Statistical tests were two-sided and a probability $(p)$ value of $<0.05$ was considered statistically significant. All analyses were performed using SPSS software version 16.0 (SPSS, Inc., Chicago, IL, USA).

\section{Results}

Concerning the baseline characteristics of two groups, no significant difference was found with respect to age, sex, BMI, fasting blood glucose, HBsAg status, Child-Pugh score, ICG15, and AFP. Surgical duration was remarkably longer in the surgery group versus the RFA group. The postoperative target area was a low-density shadow without enhancement. The complete ablation rate was $94.4 \%(51 / 53)$. In the RFA group, ablation was incomplete in three cases. Artificial hydrothorax was conducted in two cases.
Six patients underwent regular hepatic lobectomy and the other 48 underwent non-regular lobectomy. Mean blood loss in the surgery group was about $79.2 \mathrm{~mL}$, with an average Pringle time of $3.8 \mathrm{~min}$ (Table 1). Also, there was a significant difference in serum levels of TBil $(t=2.601, p=0.011)$, but not WBC, ALT, AST, and $\mathrm{Alb}$, or $\triangle \mathrm{PT}$ between the two groups before surgery (Table 2 ).

On D1, there were statistically significant differences in all of the above blood sample parameters between the two groups. However, on D3, other than Alb $(t=1.114, p=0.268)$, the serum levels of WBC, ALT, AST, and TBil, and $\triangle \mathrm{PT}$ were still increased in the surgery group. Furthermore, on D7, WBC, ALT, AST, and $\triangle \mathrm{PT}$ were still increased in the surgery group, but TBil and Alb between two groups were almost the same (TBil, $t=1.420, p=0.158$; Alb, $t$ $=0.349, p=0.727$ ).

As shown in Table 3, the prevalence of excessive pain, total complications, and total infections was significantly lower in the RFA group as compared to the surgery group $(p<0.001)$. A significant decrease in the duration of the hospital stay after treatment was also observed in the RFA group $(t=10.017, p<0.001)$. The trend seemed to favor a reduction in postoperative mortality in the RFA group (Table 3).
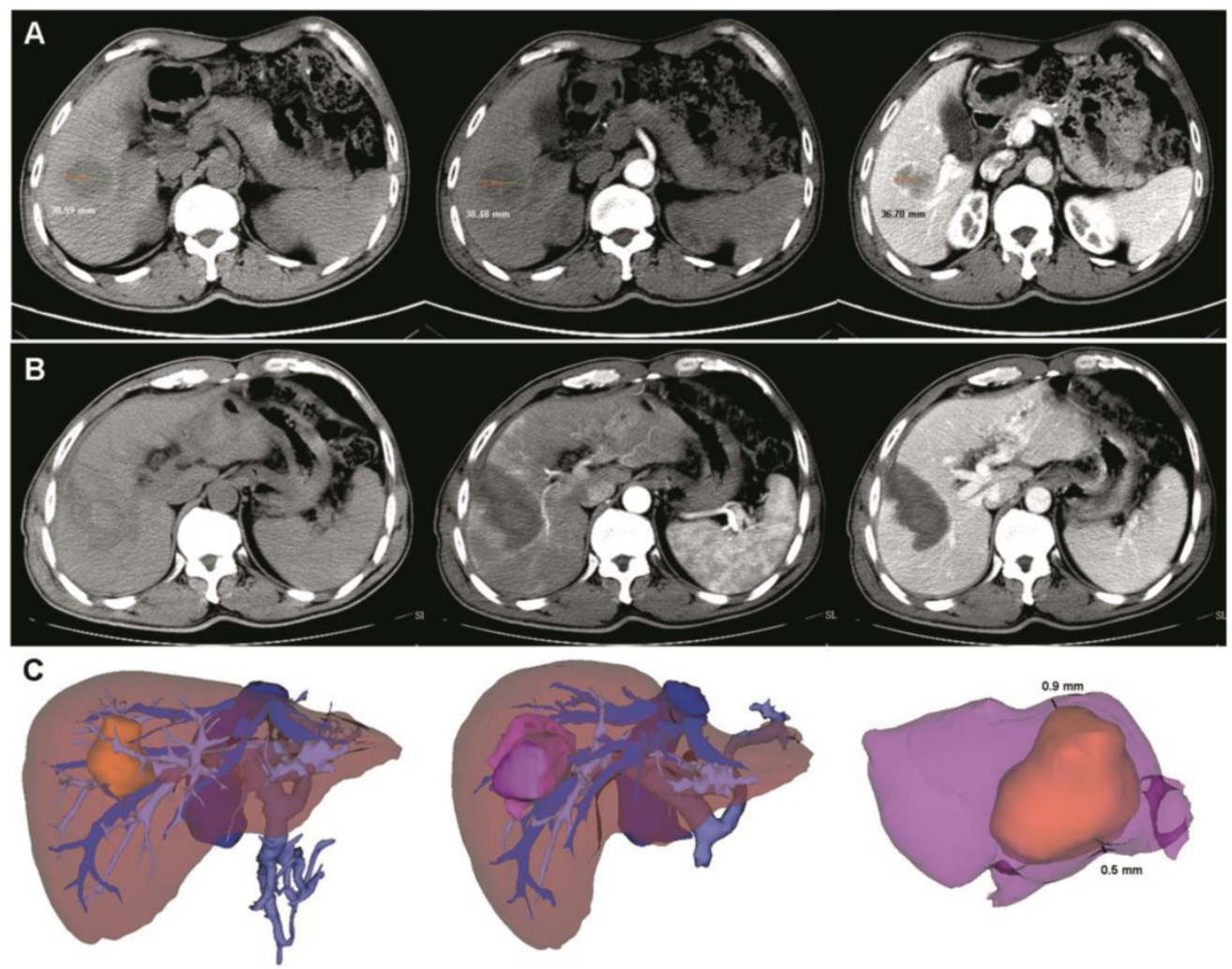

Figure 1. Pre- and post- operative 3D-CT reconstruction and image fusion. (A) Pre-operative two-dimension computed tomography (plain scan, arterial and venous phases). The tumor size was about $38.59 \mathrm{~mm}$. (B) After ultrasound-guided RFA combined with 3D-CT reconstruction, a low-density area was observed (plain scan, arterial and venous phases). (C) Pre- and post-operative 3D-CT reconstruction and image fusion. The yellow mass indicates the tumor and the purple mass indicates the ablation area. After image fusion, the safety margin was measured in a multidimensional approach. In this case, the thinnest safety margin was 0.6 mm. RFA, radiofrequency ablation. 
Table 1. Baseline characteristics of the two groups.

\begin{tabular}{|c|c|c|c|c|}
\hline & $\begin{array}{l}\text { RFA Group (\%) } \\
(n=54)\end{array}$ & $\begin{array}{l}\text { Surgery Group (\%) } \\
(n=66)\end{array}$ & $t / \chi^{2}$ & $p$ \\
\hline \multicolumn{5}{|l|}{ Age (years) } \\
\hline$\leq 50$ & $46(85.2)$ & $58(87.9)$ & 0.186 & 0.666 \\
\hline$>50$ & $8(14.8)$ & $8(12.1)$ & & \\
\hline \multicolumn{5}{|l|}{ Sex } \\
\hline Male & $38(70.4)$ & $49(74.2)$ & 0.223 & 0.637 \\
\hline Female & $16(29.6)$ & $17(25.8)$ & & \\
\hline \multicolumn{5}{|l|}{ BMI } \\
\hline$\leq 18.5$ & $8(14.8)$ & 9 (13.6) & 0.486 & 0.922 \\
\hline $18.5-22.9$ & $23(42.6)$ & $25(37.9)$ & & \\
\hline $23.0-24.9$ & $20(37.0)$ & $27(40.9)$ & & \\
\hline$>25$ & $3(5.6)$ & $5(7.6)$ & & \\
\hline FBG (mmol/L) & $4.9 \pm 1.9$ & $5.1 \pm 1.8$ & 0.591 & 0.556 \\
\hline HBsAg positive & $47(87.0)$ & $55(83.3)$ & 0.320 & 0.572 \\
\hline \multicolumn{5}{|l|}{ Child-Pugh score } \\
\hline Score A & $48(88.9)$ & $61(92.4)$ & 0.446 & 0.504 \\
\hline Score B & $6(11.1)$ & $5(7.6)$ & & \\
\hline ICG R15 (\%) & $7.54 \pm 4.83$ & $6.37 \pm 3.81$ & 1.484 & 0.141 \\
\hline \multicolumn{5}{|l|}{$\operatorname{AFP}(n g / m L)$} \\
\hline$\leq 20$ & $38(70.4)$ & $46(69.7)$ & 0.006 & 0.936 \\
\hline$>20$ & $16(29.6)$ & $20(30.3)$ & & \\
\hline $\begin{array}{l}\text { Surgical duration } \\
\text { (min) }\end{array}$ & $72.9 \pm 36.3$ & $156.3 \pm 73.1$ & 7.644 & $<0.001$ \\
\hline Complete ablation & $51(94.4)$ & & NA & NA \\
\hline Blood loss (mL) & & $79.2 \pm 39.2$ & NA & NA \\
\hline Pringle time (min) & & $3.8 \pm 1.4$ & NA & NA \\
\hline
\end{tabular}

Table 2. Blood sample tests of the two groups before and after surgery.

\begin{tabular}{|c|c|c|c|c|}
\hline & $\begin{array}{l}\text { RFA Group (\%) } \\
(n=54)\end{array}$ & $\begin{array}{l}\text { Surgery Group (\%) } \\
(n=66)\end{array}$ & $t$ & $p$ \\
\hline \multicolumn{5}{|l|}{ WBC $\left(\times 10^{9} / \mathrm{L}\right)$} \\
\hline Preoperation & $6.23 \pm 2.89$ & $6.67 \pm 3.01$ & 0.811 & 0.419 \\
\hline D1 & $13.25 \pm 5.39$ & $18.23 \pm 7.26$ & 4.184 & $<0.001$ \\
\hline D3 & $11.75 \pm 5.02$ & $14.37 \pm 6.19$ & 2.508 & 0.014 \\
\hline D7 & $7.26 \pm 3.68$ & $9.56 \pm 4.69$ & 2.938 & 0.004 \\
\hline \multicolumn{5}{|l|}{ ALT (IU/L) } \\
\hline Preoperation & $32.31 \pm 14.25$ & $28.19 \pm 12.52$ & 1.685 & 0.095 \\
\hline D1 & $274.49 \pm 129.53$ & $426.53 \pm 224.57$ & 4.409 & $<0.001$ \\
\hline D3 & $126.32 \pm 61.12$ & $264.62 \pm 127.98$ & 7.286 & $<0.001$ \\
\hline D7 & $48.91 \pm 21.38$ & $69.91 \pm 26.75$ & 4.674 & $<0.001$ \\
\hline \multicolumn{5}{|l|}{ AST (IU/L) } \\
\hline Preoperation & $34.75 \pm 16.83$ & $35.92 \pm 17.29$ & 0.373 & 0.710 \\
\hline D1 & $247.74 \pm 138.03$ & $397.75 \pm 213.75$ & 4.452 & $<0.001$ \\
\hline D3 & $108.96 \pm 53.03$ & $227.79 \pm 113.84$ & 7.065 & $<0.001$ \\
\hline D7 & $41.23 \pm 23.68$ & $56.68 \pm 28.75$ & 3.166 & 0.002 \\
\hline \multicolumn{5}{|l|}{ TBil (umol/L) } \\
\hline Preoperation & $17.33 \pm 6.76$ & $14.48 \pm 5.24$ & 2.601 & 0.011 \\
\hline D1 & $26.37 \pm 10.65$ & $33.47 \pm 17.95$ & 2.560 & 0.012 \\
\hline D3 & $18.68 \pm 8.53$ & $22.38 \pm 10.25$ & 2.119 & 0.036 \\
\hline D7 & $16.47 \pm 6.83$ & $14.57 \pm 7.65$ & 1.420 & 0.158 \\
\hline \multicolumn{5}{|l|}{$\operatorname{Alb}(g / L)$} \\
\hline Preoperation & $35.35 \pm 4.54$ & $37.39 \pm 6.47$ & 1.956 & 0.053 \\
\hline D1 & $32.75 \pm 5.47$ & $29.97 \pm 4.90$ & 2.934 & 0.004 \\
\hline D3 & $33.49 \pm 5.87$ & $32.31 \pm 5.69$ & 1.114 & 0.268 \\
\hline D7 & $35.76 \pm 6.67$ & $36.23 \pm 7.83$ & 0.349 & 0.727 \\
\hline \multicolumn{5}{|l|}{$\Delta \mathrm{PT}(\mathrm{s})$} \\
\hline D1 & $0.73 \pm 0.43$ & $0.54 \pm 0.33$ & 2.737 & 0.007 \\
\hline D3 & $0.32 \pm 0.25$ & $0.15 \pm 0.32$ & 3.188 & 0.002 \\
\hline D7 & $-0.26 \pm 0.19$ & $-0.11 \pm 0.21$ & 4.062 & $<0.001$ \\
\hline
\end{tabular}

$\triangle \mathrm{PT}$, prothrombin time, true value - upper limit of normal value; $\mathrm{Alb}$, albumin; ALT, alanine aminotransferase; AST, aspartate transaminase; D, day; RFA, radiofrequency ablation; TBil, total bilirubin; WBC, white blood cell count.
Table 3. Complications, mortality, and duration of hospital stay after surgery of the two groups

\begin{tabular}{lll}
\hline & $\begin{array}{l}\text { RFA Group (\%) } \\
(\boldsymbol{n}=\mathbf{5 4})\end{array}$ & $\begin{array}{l}\text { Surgery Group (\%) } \\
(\boldsymbol{n}=\mathbf{6 6})\end{array}$ \\
\hline Excessive pain & $13(24.1)$ & $66(100)$ \\
Total complications & $2(3.7)$ & $18(27.3)$ \\
Abdominal hemorrhage & 0 & 2 \\
Upper gastrointestinal hemorrhage & 0 & 1 \\
Biliary leakage & 0 & 1 \\
Liver failure & 0 & 2 \\
Total infection & $2(3.7)$ & $12(18.2)$ \\
Wound infection & 0 & 0 \\
Pulmonary infection & 2 & 11 \\
Abdominal infection & 0 & 1 \\
Sepsis & 0 & 1 \\
Mortality & $0(0)$ & $1(1.5)$ \\
Duration of hospital stay after & $2.65 \pm 1.79$ & $9.43 \pm 4.70$ \\
surgery & &
\end{tabular}

Of the 120 study participants, 116 (96.7\%) were followed-up by phone, and one case in the RFA group and three cases in the surgery group were lost to follow-up. During a median follow-up duration of 22.4 (range, 7.3-45.1) months, no tumor seeding was observed in the RFA group. The 1-, 2-, and 3-year recurrence rates were $18.9 \%, 45.3 \%$, and $50.9 \%$ for the RFA group, and $23.8 \%, 39.7 \%$, and $49.2 \%$ for the surgery group, respectively $(\chi 2=0.217, p=0.641)$. The corresponding OS rates were $88.7 \%, 73.6 \%$, and $66.0 \%$ for the RFA group, and $93.7 \%, 76.2 \%$, and $58.7 \%$ for the surgery group, respectively $\left(\chi^{2}=0.484, p=0.486\right)$. There was no significant difference in the cumulative recurrence rates between the two groups over the follow-up period (Figure 2). There was no significant difference in the OS rate between two groups, as determined with the log-rank test (Figure 3).

\section{Discussion}

In recent years, ultrasound-guided RFA has become an acceptable and frequently used therapeutic option for patients with small HCC. However, for patients with solitary HCC of $3-5 \mathrm{~cm}$ who are candidates for both RFA and curative surgical treatment, safety and effectiveness remain controversial. A recent randomized clinical trial conducted in Hong Kong showed that for HCC smaller than $5 \mathrm{~cm}$, or less than 3 tumors, each smaller than $3 \mathrm{~cm}$, the overall tumor recurrence rate was similar in the RFA and resection groups. The 1-, 3-, 5-, and 10 -year OS rates were $95.4 \%, 82.3 \%, 66.4 \%$, and $41.8 \%$ in the RFA group, compared with $94.5 \%$, $80.6 \%, 66.5 \%$, and $47.6 \%$, respectively, in the resection group $(p=0.531)$, indicating that long-term survival (10-year OS) was superior in the resection group [10]. A large retrospective study, for which patients were divided into groups based on tumor size (i.e., $\leq 20$, $21-30$, and $31-50 \mathrm{~mm}$ ), reported that there was no 
significant difference in OS between the HCC $\leq 20$ $\mathrm{mm}$ vs. 21-30 mm groups. However, for patients with tumors measuring $31-50 \mathrm{~mm}$, OS was poorer in the RFA group than in the resection group. Interestingly, the worse OS noted with RFA was observed with only a 5-mm increase in tumors measuring $>30 \mathrm{~mm}$ [5].

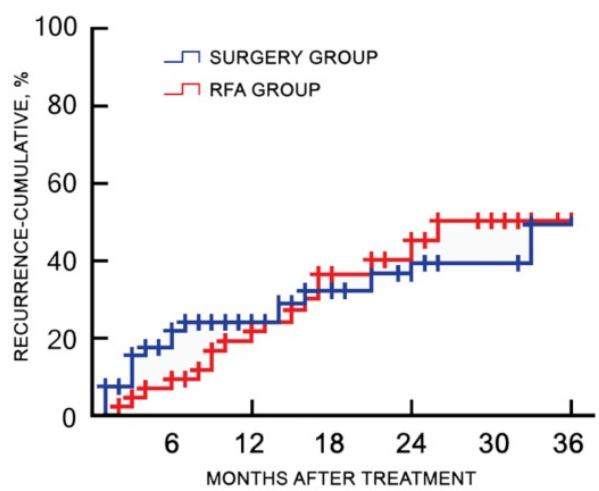

Figure 2. Cumulative recurrence rates of the RFA and surgery groups after treatment. There were no significant differences in the cumulative recurrence rate between the two groups during the follow-up period. RFA, radiofrequency ablation.

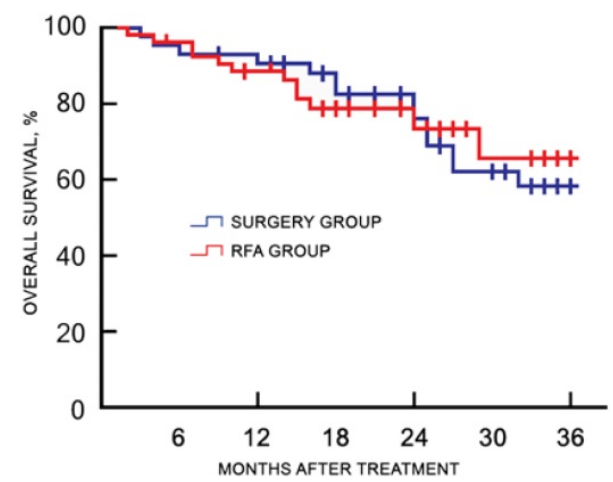

Figure 3. Kaplan-Meier plot for OS between the RFA and surgery groups after treatment. OS, overall survival; RFA, radiofrequency ablation.

In light of this controversy, we agree that it is crucial to arrive at a comprehensive RFA plan to ensure a tumor-free margin and improve the complete ablation rate. A prospective randomized trial comparing the 3-year clinical outcomes of RFA targeting 5- or $10-\mathrm{mm}$ margins demonstrated that targeting a 10-mm margin could significantly reduce the risk of tumor recurrence [6]. Similarly, multivariate analysis from previous studies also revealed that an insufficient margin (5 $\mathrm{mm}$ [11] or 3 $\mathrm{mm}$ [12]) was an independent predisposing factor for overall recurrence after RFA. Our previous study investigating the feasibility of $3 \mathrm{D}-\mathrm{CT}$ image fusion to assess the ablation margin also demonstrated that a sufficient ablation margin wider than $5 \mathrm{~mm}$ was an independent prognostic factor for both OS and PFS [9]. Based on the 3D-CT image fusion technique, the current study compared the outcomes of a new method, ultrasound-guided RFA combined with preoperative $3 \mathrm{D}$ reconstruction, with surgical resection among patients with solitary HCC of 3-5 $\mathrm{cm}$. The short-term results showed that the incidences of excessive pain, total complications, and total infections were significantly lower in the RFA group than in the surgery group. A significant decrease in the duration of the hospital stay after treatment was also observed in the RFA group. The long-term results with a 3-year follow-up period showed that there were no significant differences in the cumulative recurrence and OS rates between two groups. This result indicated that ultrasound-guided RFA combined with preoperative $3 \mathrm{D}$ reconstruction might be a safe and effective therapeutic option for patients with solitary HCC of 3-5 cm.

Conventional ultrasound-guided RFA can only be used to access the ablation margin on twodimensional images, which may contribute to incomplete ablation particularly for HCC larger than $3 \mathrm{~cm}$. Also, incomplete ablation and sublethal heat treatment could skew HCC cells toward epithelialmesenchymal transition (EMT) and transform them to a progenitor-like, highly proliferative cellular phenotype both in vitro and in vivo, which is driven significantly by p46Shc-Erk1/2 [13]. In addition, incomplete ablation enhanced the invasive and metastatic potential of residual cancer, accompanied with EMT-like phenotype changes by activating $\beta$-catenin signaling in HCCLM3 cells [14]. Sorafenib reportedly can inhibit the EMT of HepG2 and SMMC7721 cells after insufficient RFA, and might be useful to prevent the progression of HCC after RFA [15]. In the present study, ultrasound-guided RFA combined with preoperative 3D reconstruction provided intuitional images of the tumor location, tumor size, and adjacent vasculature in all directions to obtain a comprehensive plan. Afterward, precision and the complete ablation rate can be improved by inserting the electrode needle, while avoiding repeating punctures. Another improved technique, real-time ultrasonography (US)/CT-MRI image fusion-guided RFA, has become increasingly accepted worldwide. A pilot study showed that for HCC smaller than $5 \mathrm{~cm}$, US/CT-MRI image fusion improved tumor visibility and the technical feasibility of RFA. Fusion imaging-guided RFA using multiple electrodes demonstrated a highly effective ablation rate and a low local tumor progression rate during a 2-year follow-up period [16-17].

In the current study, the rate of excessive pain in the RFA group was notably lower than in the surgery group, and the incidence of postoperative complications in RFA group was about $3.7 \%$. No serious complications, such as liver failure, was 
observed. Surgical experience is an important factor that influences the surgical outcome, but it is immeasurable in ways as clear as, for example, complications are measured in patients [18]. The learning curve of hepatectomy is dramatically longer than that of ultrasound-guided RFA. The preoperative $3 \mathrm{D}$ reconstruction can help to shorten the learning curve of both RFA and hepatectomy, thereby avoiding artificial influences and experiential differences between different surgeons. Hence, further investigations with larger sample sizes and well-controlled for confounding factors are needed to confirm the long-term effect of ultrasound-guided RFA combined with preoperative 3D reconstruction for solitary HCC of 3-5 cm, so that more surgeons can choose this technique as a first-line or alternative treatment for solitary HCC of 3-5 cm.

\section{Conclusion}

As compared with surgical resection for solitary HCC of 3-5 cm, ultrasound-guided RFA combined with preoperative $3 \mathrm{D}$ reconstruction could significantly reduce the morbidity of excessive pain, total complications, and infections, as well as shorten the duration of the hospital stay after treatment. No significant differences were observed in the cumulative recurrence and OS rates during a 3-year follow-up period. Ultrasound-guided RFA combined with preoperative $3 \mathrm{D}$ reconstruction appears to be a safe and effective therapeutic option for patients with solitary HCC of 3-5 cm.

\section{Acknowledgments}

We acknowledge Hao Lu for his help in threedimensional reconstruction and image fusion.

\section{Financial Support}

This work was supported by grants from the Guangdong Medical Research Project (No.A2018247), Guangzhou Health and Family Planning Technology Project (No.20191A011096) awarded to Y.G., and the National Natural Science Foundation of China (No. 81602172), Guangdong Provincial Science and Technology Plan projects (No.2016A030313769), Guangzhou Science and Technology Plan of Scientifc Research Projects (No.201707010323) awarded to B.H.

\section{Author Contributions}

Yuanfeng Gong and Baohua Hou conceived and designed the study. Yu Zhou and Min Yu identified and included eligible subjects. Bowen Huang, Zhonghai Sun and Zhixiang Jian extracted data from electronic medical records. Yunqiang Tang performed the 3D reconstruction and image fusion. Yuanfeng Gong carried out the statistical analysis and interpreted the results. The authors have no possible conflicts of interest to disclose. All drafts of the reports were written by Yuanfeng Gong and Baohua Hou. All authors read and approved the final version of the manuscript.

\section{Competing Interests}

The authors have declared that no competing interest exists.

\section{References}

1. Torre LA, Bray F, Siegel RL, Ferlay J, Lortet-Tieulent J, Jemal A. Global cancer statistics, 2012. CA Cancer J Clin. 2015; 65: 87-108.

2. Athanasopoulos P, Mastoraki A, Papalois A, Nastos C, Kondi-Pafiti A, Kostopanagiotou G, et al. Expression of Inflammatory and Regenerative Genes in a Model of Liver Ischemia/Reperfusion and Partial Hepatectomy. J Invest Surg. 2016; 29: 67-73.

3. Zhang L, Ge NL, Chen Y, Xie XY, Yin X, Gan YH, et al. Long-term outcomes and prognostic analysis of radiofrequency ablation for small hepatocellular carcinoma: 10-year follow-up in Chinese patients. Med Oncol. 2015; 32: 77.

4. Fang Y, Chen W, Liang X, Li D, Lou H, Chen R, et al. Comparison of long-term effectiveness and complications of radiofrequency ablation with hepatectomy for small hepatocellular carcinoma. J Gastroenterol Hepatol. 2014; 29: 193-200.

5. Kutlu OC, Chan JA, Aloia TA, Chun YS, Kaseb AO, Passot G, et al. Comparative effectiveness of first-line radiofrequency ablation versus surgical resection and transplantation for patients with early hepatocellular carcinoma. Cancer. 2017; 123: 1817-27.

6. Liao M, Zhong X, Zhang J, Liu Y, Zhu Z, Wu H, et al. Radiofrequency ablation using a 10-mm target margin for small hepatocellular carcinoma in patients with liver cirrhosis: A prospective randomized trial. J Surg Oncol. 2017; 115: 971-9.

7. Morise Z, Kawabe N, Tomishige H, Nagata H, Kawase J, Arakawa S, et al. Recent advances in liver resection for hepatocellular carcinoma. Front Surg. 2014; 1: 21.

8. Memeo R, De'Angelis N, de Blasi V, Cherkaoui Z, Brunetti O, Longo V, et al. Innovative surgical approaches for hepatocellular carcinoma. World J Hepatol. 2016; 8: 591-6.

9. Tang H, Tang Y, Hong J, Chen T, Mai C, Jiang P. A measure to assess the ablative margin using 3D-CT image fusion after radiofrequency ablation of hepatocellular carcinoma. HPB (Oxford). 2015; 17: 318-25.

10. Ng K, Chok K, Chan A, Cheung TT, Wong T, Fung J, et al. Randomized clinical trial of hepatic resection versus radiofrequency ablation for early-stage hepatocellular carcinoma. Br J Surg. 2017; 104: 1775-84.

11. Zytoon AA, Ishii H, Murakami K, El-Kholy MR, Furuse J, El-Dorry A, et al. Recurrence-free survival after radiofrequency ablation of hepatocellular carcinoma. A registry report of the impact of risk factors on outcome. Jpn J Clin Oncol. 2007; 37: 658-72.

12. Toshimori J, Nouso $K$, Nakamura S, Wada N, Morimoto $Y$, Takeuchi $Y$, et al. Local recurrence and complications after percutaneous radiofrequency ablation of hepatocellular carcinoma: a retrospective cohort study focused on tumor location. Acta Med Okayama. 2015; 69: 219-26.

13. Yoshida S, Kornek M, Ikenaga N, Schmelzle M, Masuzaki R, Csizmadia E, et al. Sublethal heat treatment promotes epithelial-mesenchymal transition and enhances the malignant potential of hepatocellular carcinoma. Hepatology. 2013; 58: 1667-80.

14. Zhang N, Wang L, Chai ZT, Zhu ZM, Zhu XD, Ma DN, et al. Incomplete radiofrequency ablation enhances invasiveness and metastasis of residual cancer of hepatocellular carcinoma cell HCCLM3 via activating beta-catenin signaling. PLoS One. 2014; 9: e115949.

15. Dong S, Kong J, Kong F, Kong J, Gao J, Ji L, et al. Sorafenib suppresses the epithelial-mesenchymal transition of hepatocellular carcinoma cells after insufficient radiofrequency ablation. BMC Cancer. 2015; 15: 939.

16. Li K, Su Z, Xu E, Huang Q, Zeng Q, Zheng R. Evaluation of the ablation margin of hepatocellular carcinoma using CEUS-CT/MR image fusion in a phantom model and in patients. BMC Cancer. 2017; 17: 61.

17. Ahn SJ, Lee JM, Lee DH, Lee SM, Yoon JH, Kim YJ, et al. Real-time US-CT/MR fusion imaging for percutaneous radiofrequency ablation of hepatocellular carcinoma. J Hepatol. 2017; 66: 347-54.

18. Cataneo DC. How is a surgeon's experience measured? J Thorac Cardiovasc Surg. 2015; 149: 1005 